\title{
Skeletal and Sexual Maturation and Growth in Tokyo Girls: Longitudinal Observations
}

\author{
Kumi Ashizawa \\ Laboratory of Growth and Ergonomics, Institute of Human Living Sciences, \\ Otsuma Women's University, Tokyo, Japan
}

Key words: growth, skeletal maturation, menarche, Tokyo girls, longitudinal observations

For the study of adolescent growth observation of data obtained from longitudinal surveys is indispensable, but in practice it is almost impossible because this type of study demands enormous patience of both research workers and subject children.

In the field of biological anthropology in Japan two important longitudinal growth study projects have been carried out. One was a study of mixed Japanese-American children born of Japanese women and occupation soldiers just after the Second World War. The semi-longitudinally analyzed results have been reported up to the present. The other is the study of twins surveyed from 1974 through 1991 , of which the results are now being analyzed.

For the study of skeletal maturation, some cross-sectional studies based upon the Tanner-Whitehouse method must be

Correspondence: Kumi Ashizawa, M.D., Ph.D., Loboratory of Growth and Ergonomics, Institute of Human Living Sciences, Otsuma Women's University, 12, Sanban-cho, Chiyoda-ku, Tokyo 102 Japan

Source of support: Grant in Aid for General Scientific Research, the Ministry of Education, Science, and Culture, 1990 and 1991. mentioned. The TW1 system, assessing morphological changes of bones appearing in the left hand and wrist on X-ray film, was applied first in Japan in 1968 and 1969 to Tokyo children aged 6 to 18 (Ashizawa, 1970). They were found to present one to one-and-a-half years advanced skeletal maturation over the British children's standard.

With the TW2 method, i.e., revised TW1 rating system, children in Okinawa, Sapporo, Nagasaki, Amami-Ohshima, and Nomozaki were assessed in the 1970s and 1980s (Kimura, 1977 a, b; Takai and Akiyoshi, 1983; Takai, et al., 1984; Takai, 1990). Of these, the last one was longitudinally surveyed, but the results were reported semi-longitudinally.

Recently, radiation exposure regulations have become increasingly strict, and it has become almost impossible to survey skeletal maturation in healthy children. On the other hand, and contradictorily, data on normal healthy children's growth and maturation are being demanded by physicians according to the growing importance of treatment of shortstature children resulting from various conditions such as chromosomal aberrations, endocrinological abnormalities, and so on. 


\section{Materials}

The subject girls were selected from the data set of Eto and Ashizawa (1992). In this data set, TW2 X-ray observations conducted for more than 10 years from 1969 on 65 girls are provided with stature, body weight, and chest circumference. The subject girls were pupils in a group of private schools operated by a Buddhist temple whose families lived on the west side of Tokyo and were of an average socio-economic level in the capital. All of the girls were born between 1963 and 1966 .

Comparing contemporary standard growth curves of Tokyo girls, which were published by the Ministry of Education, Science, and Culture in Japan, no statistical difference is observed between our subject group and the standard group in stature, chest circumference and body weight.

Among these 65 subjects, 44 were selected for this study because of their correctly recorded menarcheal date. Two girls did not show any peak in their weight or chest circumference velocity curves, so that we excluded them from this study of adolescent growth, thus reducing the subject number to 42 .

\section{Methods}

\section{(1) Growth data}

To annually measured stature, body weight, and chest circumference growth curves of each girl the smoothing spline curve was applied. Smoothing spline velocity curves of these measurements were obtained as well for each individual girl with the age, size intensity, and size at the peak.

\section{(2) Skeletal maturation}

Skeletal maturation was assessed according to the TW2 rating system. To the RUS, Carpal, and 20-Bone skeletal maturity score curves and the annual velocity score curves of
Table 1. Means and standard deviations of menarcheal age, age at peak velocity, size intensity and size at the peak, and size at menarche of stature, body weight and chest circumference

\begin{tabular}{l|rrr}
\hline & $\mathrm{N}$ & Means & SD \\
\hline Menarcheal age (years) & 42 & 12.36 & 0.98 \\
\hline Age at PHV (years) & 42 & 11.06 & 0.93 \\
PHV (cm) & 42 & 7.76 & 1.20 \\
Stature at PHV (cm) & 42 & 141.62 & 6.42 \\
Stature at menarche (cm) & 42 & 150.26 & 6.39 \\
\hline Age at PWV (years) & 42 & 11.79 & 1.32 \\
PWV (kg) & 42 & 6.47 & 1.82 \\
Weight at PWV (kg) & 42 & 38.97 & 6.81 \\
Weight at menarche (kg) & 42 & 41.75 & 6.77 \\
\hline Age at PCV (years) & 42 & 11.81 & 1.19 \\
PCV (cm) & 42 & 5.44 & 1.30 \\
Chest circumference & 42 & 71.19 & 5.05 \\
$\quad$ at PCV (cm) & & & \\
Chest circumference & 42 & 73.60 & 5.53 \\
$\quad$ at menarche (cm) & & & \\
\hline
\end{tabular}

each girl, the smoothing spline curve was applied. Age, score intensity, and score at the peak were obtained.

\section{(3) Menacheal age}

The diary method was utilized. Specifically, at the beginning of this study, the mothers were asked in advance to record the date of menarche of their daughters at such time it should occur. The date was accurately recorded for the 44 girls, so that it was possible to record the correct menarcheal age.

\section{Results}

Table 1 presents the means and standard deviations of menarcheal age, age at peak velocity, size intensity and size at the peak, and size at menarche of stature, body weight and chest circumference.

Table 2 shows the means and standard deviations of age at peak score velocity, score intensity and score at the peak, and score at menarche of RUS, Carpal, and 20-Bone maturity scores.

We were able to summarize the order of the ages of peak velocity of three measure- 
ments, those of the three bone maturity scores, and menarcheal age. Menarche occurs at 12 years and 3 or 4 months of age (age 12.36). This is almost the same as the mean menarcheal age of Tokyo girls (12.3 years) but slightly precocious for Japan (12.5 years) as estimated from the status quo data of 1987 (Hinobayashi, 1990). RUS-score-peakvelocity appears closest to the menarcheal age, 11 years and 10 or 11 months; namely 4 or 5 months previous to menarche. Statural peak is attained 1 year and 3 months before menarche. Carpal-score-peak-velocity appears precociously, at age 9 .

Table 2. Means and standard deviations of age at peak score velocity, score intensity and score at the peak, and score at menarche of RUS, Carpal and 20-Bone maturity scores

\begin{tabular}{l|rrr}
\hline & $\mathrm{N}$ & Means & $\mathrm{SD}$ \\
\hline Age at RUS-score-peak- & 39 & 11.86 & 1.38 \\
$\quad$ velocity (years) & 39 & 128.2 & 23.3 \\
RUS-score-peak-velocity & 39 & 702.3 & 119.0 \\
Score at RUS-score- & 39 & & \\
$\quad$ peak-velocity & 42 & 752.0 & 77.2 \\
RUS-score at menarche & 33 & 9.02 & 1.26 \\
\hline Age at Carpal-score-peak- & 33 & & \\
$\quad$ velocity (years) & 33 & 118.3 & 34.9 \\
Carpal-score-peak-velocity & 33 & 748.8 & 137.0 \\
Score at Carpal-score- & 33 & & \\
$\quad$ peak-velocity & 42 & 987.3 & 15.4 \\
Carpal-score at menarche & 33 & 9.53 & 1.16 \\
\hline Age at 20-Bone-score- & 33 & & \\
$\quad$ peak velocity (years) & 33 & 118.6 & 25.0 \\
20-Bone-score-peak-velocity & 33 & 732.5 & 91.2 \\
Score at 20-Bone-score- & 33 & & \\
$\quad$ peak-velocity & 42 & 950.0 & 27.6 \\
20-Bone-score at menarche & & & \\
\hline
\end{tabular}

Table 3 presents statistically significant correlation coefficients concerning menarcheal age, stature, and RUS maturity score.

We can summarize these correlations as follows:

1) Girls experiencing an earlier menarcheal age show peak-height-velocity and RUSscore-peak-velocity with larger magnitude (intensity) at earlier ages, and are of a lower stature and smaller RUS-score at menarche.

2) Girls experiencing an earlier age at peak-height-velocity are of a lower stature at peak-height-velocity, but are of a larger magnitude (intensity) and earlier age at RUSscore-peak-velocity.

3) Girls having a larger peak-heightvelocity show RUS-score-peak-velocity at an earlier age.

4) Girls having a taller stature at peakheight-velocity are of a taller stature at menarche.

5) Girls having a taller stature at menarche show a larger RUS-score at menarche.

6) Girls experiencing RUS-score-peakvelocity at an earlier age show a smaller score, but of a larger magnitude.

7) Girls having a larger RUS-score-peakvelocity show a smaller score at their peak velocity.

\section{References}

1. Ashizawa, K., Maturation osseuse des enfants japonais de 6 à 18 ans, estimée par

Table 3. Statistically significant correlation coefficients concerning menarcheal age, stature, and RUS maturity score

\begin{tabular}{|c|c|c|c|c|c|c|c|}
\hline & $\begin{array}{c}\text { Menacheal } \\
\text { age }\end{array}$ & $\begin{array}{l}\text { Age at } \\
\text { PHV }\end{array}$ & PHV & $\begin{array}{c}\text { Stature at } \\
\text { PHV }\end{array}$ & $\begin{array}{l}\text { Stature at } \\
\text { menarche }\end{array}$ & $\begin{array}{l}\text { Age at RUS- } \\
\text { score peak }\end{array}$ & $\begin{array}{l}\text { RUS-score } \\
\text { p.velocity }\end{array}$ \\
\hline $\begin{array}{l}\text { Age at PHV } \\
\text { PHV } \\
\text { Stature at PHV } \\
\text { Stature at menarche } \\
\text { Age at RUS-score-peak } \\
\text { RUS-score-peak-velocity } \\
\text { Score at RUS-score-peak } \\
\text { RUS-score at menarche }\end{array}$ & $\begin{array}{l}.687^{* * *} \\
-.354^{*} \\
\\
.331^{*} \\
.350^{*}\end{array}$ & $\begin{array}{l}. .389^{*} \\
.414^{* *} \\
.508^{* * *} \\
.561^{* * *}\end{array}$ & $-.354^{*}$ & $.764^{* * *}$ & $.360^{*}$ & $\begin{array}{l}-.333^{*} \\
.835^{* * *}\end{array}$ & $-.364^{*}$ \\
\hline
\end{tabular}


la méthode de Tanner-Whitehouse. Bull Mém. Soc. Anthrop. Paris, 1970; 6(série XII) : 265-280.

2. Eto, M., Ashizawa, K., Longitudinal Observations on Physical Growth and TW2 Skeletal Maturation of Girls in Tokyo. Radiographic Atlas of Hand and Wrist. Tokyo: Terapeia, 1992.

3. Hinobayashi, T., The age of menarche in Japanese girls, in 1987. Obstetrical and Gynecological Therapy, 1990; 60: 145-149.

4. Kimura, K., Skeletal maturation of children in Okinawa, Ann. Human Biol., 1976; 3: 149-155.

5. Kimura, K., Skeletal maturity of the hand and wrist in Japanese children by the TW II method. Ann. Human Biol., 1977a; 4: 353-356.
6. Kimura, K., Skeletal maturity of the hand and wrist in Japanese children in Sapporo by the TW II method. Ann. Human Biol., 1977b; 5: 449-453.

7. Takai, S., Akiyoshi, T., Skeletal maturity of Japanese children in Western Kyushu. Am. J. Phys. Anthrop., 1983; 62: 199-204.

8. Takai, S., Akiyoshi, T., Fuchigami, A., Skeletal maturity of Japanese children in Amami-Oshima Island. Ann. Human Biol., 1984; 11: 571-575.

9. Takai, S., Smoothed skeletal maturity curve of Japanese children by TannerWhitehouse 2 (TW2) method and its application. Acta Anatomica Nipponica, 1990; 65: 436-447. (Text in Japanese with English summary.) 